\title{
Archives
}

BENNICK, A. et SITTON, J. V. (2014). Managing Active Business

Records ( 2 édition). Overland Park, KS : ARMA International, 248 pages

\section{Geneviève Piché}

Volume 47, numéro 1, 2017

URI : https://id.erudit.org/iderudit/1041833ar

DOI : https://doi.org/10.7202/1041833ar

Aller au sommaire du numéro

Éditeur(s)

Association des archivistes du Québec (AAQ)

ISSN

0044-9423 (imprimé)

2369-9256 (numérique)

Découvrir la revue

Citer ce compte rendu

Piché, G. (2017). Compte rendu de [BENNICK, A. et SITTON, J. V. (2014).

Managing Active Business Records ( ${ }^{\mathrm{e}}$ édition). Overland Park, KS : ARMA

International, 248 pages]. Archives, 47(1), 199-202.

https://doi.org/10.7202/1041833ar d'utilisation que vous pouvez consulter en ligne.

https://apropos.erudit.org/fr/usagers/politique-dutilisation/ 


\section{COMPTE RENDU}

\section{BENNICK, A. et SITTON, J. V. (2014). Managing Active Business Records ( $2^{\mathrm{e}}$ édition). Overland Park, KS: ARMA International, 248 pages.}

\section{GENEVIÈVE PICHÉ}

Historienne et archiviste responsable du centre d'archives, Le Monastère des Augustines

En 1989, Ann Bennick publia un ouvrage sur le classement des documents actifs qui a fait figure d'autorité pendant plus d'une décennie: Active Filing for Business Records. Réédité en 2000, l'ouvrage accorde alors plus d'attention aux documents électroniques et à leur gestion. Depuis, plus de dix années sont passées et l'auteure a entrepris, avec la collaboration de Judy Vasek Sitton, de mettre à jour les connaissances en la matière, notamment en ce qui concerne les informations conservées sous forme électronique (ESI) et les questions d'ordre juridique et de règlementation. L'ouvrage reprend sensiblement le même modèle; quatorze chapitres se succèdent, avec une riche série d'illustrations, de cas d'études, de tableaux, de formulaires et d'annexes.

Dès les premières pages du premier chapitre, les auteures exposent la structure de l'ouvrage et explicitent son double objectif: celui d'explorer 
les concepts, les principes et les processus qui guident la gestion documentaire et celui d'analyser sur le développement, l'implantation et le maintien d'un système de classement et de classification de documents actifs. Une fois ces bases établies, le chapitre deux s'attarde sur ce que comprend un tel système, surtout devant «l'explosion d'informations et de documents» (p. 5) propre à l'ère actuelle. Les auteures insistent sur l'importance de développer un système de classement, un système de classification et une taxonomie pertinents - basés entre autres sur les recommandations des usagers, le cycle de vie des documents et les besoins légaux de l'entreprise - et d'élaborer des index. Le chapitre trois résume quant à lui les sept étapes préalables à l'implantation d'un système de classement et de classification de documents actifs, de l'analyse des besoins à l'implantation et à son suivi, en passant par la recherche de stratégies et les coûts et bénéfices estimés.

Dans le chapitre quatre, les auteures détaillent ce qu'implique la mise en œuvre d'un système de classement et de classification des documents, notamment par le biais d'entrevues avec les usagers et par l'inventaire des dossiers. Les différentes façons d'inventorier la masse documentaire sont d'ailleurs décrites de manière plus approfondie dans le chapitre cinq, alors que les auteures conseillent de procéder à un échantillonnage et de réserver l'inventaire complet aux documents de haute valeur ou qui sont appelés à être conservés à long terme. Le chapitre six se consacre pour sa part à l'analyse des enquêtes et de l'inventaire, en prenant en compte les informations préliminaires obtenues et, surtout, la fréquence d'utilisation et le degré d'accessibilité des différents documents. Le chapitre propose différents modèles tant pour le système de classement que pour le système de classification avec codes. Dans le chapitre sept, les auteures rappellent l'utilité d'avoir sous la main des procédures écrites, depuis les étapes préliminaires d'implantation, avec les enquêtes et les inventaires, jusqu'à la création d'index et de manuels, et de les rendre disponibles aux usagers.

Le chapitre huit est dédié aux documents électroniques et au souci de préservation qui en découle. La conversion, la migration, le contrôle des versions et la numérisation y sont tour à tour décrits et analysés. Les auteures ouvrent ici une parenthèse sur l'importance de cataloguer tous les documents électroniques d'une organisation (le data mapping), d'établir une marche à suivre pour conserver les informations des organisations, des employés ou des systèmes antérieurs, ainsi que de 
mettre en place des politiques pour la gestion des courriels. Le chapitre neuf se concentre quant à lui sur les questions légales et règlementaires et revient sur l'importance de retrouver rapidement les informations demandées, notamment par le biais des métadonnées dans le cas des documents électroniques. Les auteures soulèvent les questions des accès limités et sécurisés pour certains documents, en regard de diverses lois, et analysent les méthodes de destruction des documents.

Les chapitres dix et onze introduisent l'équipement nécessaire au classement des documents. La sélection doit se faire selon plusieurs facteurs: la capacité de rangement des espaces d'entreposage, les ressources financières de l'organisation, la sécurité requise pour les documents, l'usage et la fréquence d'utilisation des documents, les besoins de rangement à plat ou vertical. Une section est également consacrée aux logiciels et à l'équipement requis pour les documents électroniques. L'équipement choisi doit être facilement accessible pour les usagers et assurer un environnement sécuritaire. Le chapitre douze explore pour sa part les différentes fournitures d'entreposage, notamment les différents modèles de dossiers et d'onglets. Encore une fois, la sélection doit être effectuée selon différents facteurs: le style souhaité, I'usage des documents, la taille et le format des documents, leur fréquence d'utilisation, leur durée de vie, le volume des documents à entreposer et les ressources financières de l'organisation.

Une fois l'équipement et les fournitures sélectionnés, la mise en place du système peut commencer. Le chapitre treize souligne d'abord la nécessité d'obtenir l'approbation des dirigeants de l'organisation avant de commencer l'implantation et de procéder à la conversion des documents papier et électroniques. Le chapitre quatorze traite enfin du maintien du système, notamment en ce qui concerne la formation des usagers, la nécessité de procéder aux mises à jour des logiciels utilisés, de tenir à jour une documentation pertinente et de maintenir l'intégrité du système de classification.

Ambitieux, cet ouvrage vise à permettre à tout novice de prendre connaissance des bases en gestion documentaire, en vue de se construire un plan d'action afin de développer, d'implanter et d'entretenir un système de traitement des documents actifs. À première vue, I'ambition paraît démesurée. Traiter tous les aspects qui sous-tendent cet imposant domaine en seulement 260 pages est un pari osé. Par manque d'espace 
ou de temps, les auteures ne font bien souvent qu'en cerner rapidement le sujet, sans toujours entrer dans les détails pratiques. Les encadrés, les graphiques et les annexes permettent toutefois d'avoir quelques exemples de cas réels qui répondent souvent aux interrogations du lecteur. Par ailleurs, les chapitres s'imbriquent aisément les uns dans les autres, ce qui permet de bien comprendre les étapes successives que requiert l'implantation d'un tel système. Et fort heureusement, la section "Lectures suggérées et autres sources" pallie le manque de profondeur de l'ouvrage, en dirigeant le lecteur vers des travaux et des liens Internet pertinents. Dans l'ensemble, l'ouvrage constitue un outil simple et convivial, fort utile et accessible à tous, qui devrait se retrouver dans la bibliothèque de toute institution qui se lance dans le traitement et le classement de ses documents actifs.

En conclusion, l'étude de Bennick et Sitton demeure encore aujourd'hui une référence majeure pour l'ensemble de la profession archivistique, malgré les différences législatives qui distinguent les États-Unis du Canada. Tout y est parfaitement décrit et illustré et les nombreux cas d'étude ne font que mieux prouver les dires des auteures. II ne reste qu'à souhaiter une traduction française qui pourrait servir tant aux professionnels francophones qu'aux étudiants en archivistique du Québec.

GeneVièVe Piché 